EPJ Web of Conferences 28, 07005 (2012)

DOI: $10.1051 /$ epjconf/20122807005

(C) Owned by the authors, published by EDP Sciences, 2012

\title{
Searches for Low Mass Higgs Boson at the Tevatron
}

\author{
Federico Sforza ${ }^{\mathrm{a}}$ for the CDF and D0 Collaborations \\ University \& INFN Pisa
}

\begin{abstract}
We present the result of the searches for a low mass Standard Model Higgs boson performed at the Tevatron $p \bar{p}$ collider $(\sqrt{s}=1.96 \mathrm{TeV})$ by the CDF and D0 experiments with an integrated luminosity of up to $8.5 \mathrm{fb}^{-1}$. Individual searches are discussed and classified according to their sensitivity. Primary channels rely on the associate production with a vector boson $(W H$ or $Z H$ ) and the $H \rightarrow b \bar{b}$ decay channel (favored for $M_{H} \lesssim 135 \mathrm{GeV} / c^{2}$ ). Event selection is based on the leptonic decay of the vector boson and the identification of $b$-hadron enriched jets. Each individual channel is sensitive, for $M_{H}=115 \mathrm{GeV} / c^{2}$, to less than 5 times the $\mathrm{SM}$ expected cross section and the most sensitive channels can exclude a production cross section of $2.3 \times \sigma_{H}^{S M}$ Secondary channels rely on a variety of final states. Although they are from 2 to 5 times less sensitive than any primary channel, they contribute to the Tevatron combination and, in some cases, they pose strong constrains on exotic Higgs boson models.
\end{abstract}

\section{Introduction}

The spontaneous symmetry breaking mechanism [1] offers a possible explanation for $W$ and $Z$ boson mass within the Standard Model [2] of particle physics. A new scalar particle, the Higgs boson, is predicted but direct experimental confirmation is missing.

In this paper we summarize the direct searches performed at the Tevatron $p \bar{p}$ collider $(\sqrt{s}=1.96 \mathrm{TeV})$ by the CDF and D0 experiments with the summer 2011 dataset, corresponding to an integrated luminosity of up to $8.5 \mathrm{fb}^{-1}$. Analyses are optimized for the low range of allowed Higgs boson masses: $100 \lesssim M_{H} \lesssim 135 \mathrm{GeV} / c^{2}$. This range is favored by indirect constraints coming from the measurement of other SM parameters [3].

\section{Low Mass Higgs Analyses}

The hadron collider environment is experimentally complex because of the overwhelming background of multijet events hiding rare processes such as Higgs boson production. We need a distinct event signature to increase the signal over background ratio and thus the sensitivity. The individual analyses can be classified in two classes on the basis of the final states and the expected sensitivity:

Primary Channels: they identify the most sensitive analyses and they all share common characteristics. The Higgs boson is produced in association with a $W$ or a $Z$ bosons (see Figure 1 for predicted cross sections) and the leptonic decay of the vector boson is used for the online and offline event selection. The Higgs candidates are selected in the $b \bar{b}$ final state, as Figure 2 shows, this is the favored final state (for $M_{H} \lesssim$ $135 \mathrm{GeV} / c^{2}$ ) because of the Yukawa coupling of the SM Higgs boson to the fermions [1]. More details about the analysis techniques are given (Section 3.1) because of the relevant impact of these analyses. Article available at $n$ ttp://www.epj-conterences.org or

${ }^{a}$ e-mail: federico.sforza@pi.infn.it

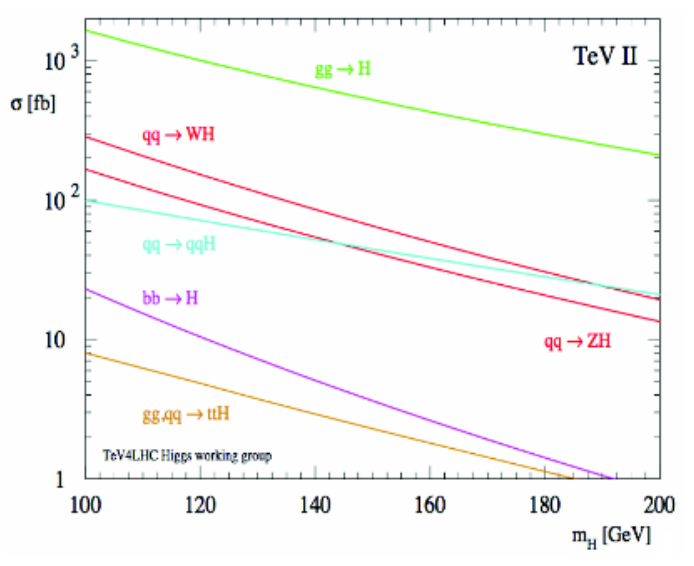

Fig. 1. Cross section of the different processes contributing to the SM Higgs boson production at the Tevatron $p \bar{p}$ collider $(\sqrt{s}=$ $1.96 \mathrm{TeV}$ ) as a function of $M_{H}$.

Secondary Channels: the Higgs is produced via gluon fusion or $t \bar{t}$ associate production (see Figure 1). Each analysis is optimized for a different final state appearing in Figure 2: $\gamma \gamma, \tau \tau$, high $b$-jets multiplicity. Each secondary channel analysis is from 2 to 5 times less sensitive than any primary channel but their contribution is not negligible when considered all together. Furthermore, many non SM Higgs boson scenarios predict a production rate which increase in these final states.

\section{Primary Search Channels}

All primary channels analyses look for the $H \rightarrow b \bar{b}$ decay when produced in association with a $W$ or $Z$ boson that undergo a leptonic decay. They are classified by the following signatures:

or http://ax.doi.org/10.1051/epjcont/20122807005




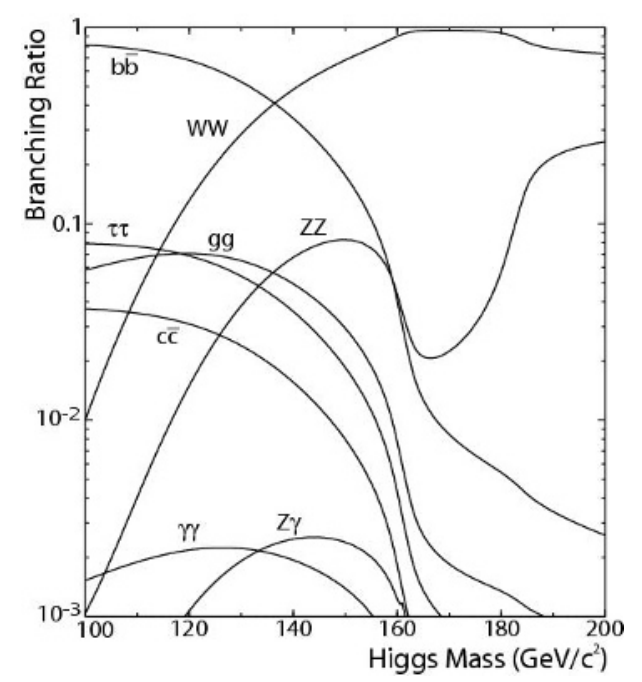

Fig. 2. SM Higgs boson branching fractions depending on the considered $M_{H}$.

where $\ell$ is an electron or a muon and $\ell$ indicates a neutrino or a lepton which has not been identified (e.g. $W \rightarrow \tau v$ events) appearing as an imbalance in the total transverse energy $\left(E_{T}\right)$. Both CDF and D0 collaborations performed analyses requiring two leptons $[4,5]$, one lepton plus $E_{T}^{\prime}[6$, 7] and $E_{T}$ only $[8,9]$. Although higher lepton multiplicity usually corresponds to a cleaner signature, the channels with one or no lepton identified are slightly more sensitive because of the higher selection efficiency and $\mathrm{WH}$ production cross section (see Figure 1).

Background composition is another common feature of the three primary channels both in CDF and D0 analyses, we can divide it in three categories: the larger is the $W / Z$ production in association with light and heavy flavor jets, after selection this irreducible background can be from $10^{2}$ to $10^{4}$ larger than the expected signal. The second background is due to multi-jet events faking the $E_{T}$ and the lepton identification. The contribution of real physics processes and detector effects makes this background particularly difficult to model so the contamination should be reduced as much as possible at selection level. The last category is composed by smaller electroweak processes like top-quark or diboson production.

Because of the small Higgs production cross section, the final challenge is the maximization of the acceptance while keeping the backgrounds under control. Single-top observation $[10,11]$ and diboson evidence in the heavy flavor final states $[12,13]$ already demonstrate that the Tevatron experiments can probe sub-picobarn cross sections in these channels.

\subsection{Analysis Techniques}

The analysis process of the primary channels can be divided into four stages: online selection, offline lepton selection, application of b-tagging algorithm and evaluation of the final discriminant. In the CDF and D0 analyses, each of these stages have been highly optimized, often thanks to the use of multivariate techniques: Neural Networks (NN), Boosted Decision Trees (BDT) or Support Vector Machines (SVM) [14]. Machine learning algorithms are powerful re- gression or classification tools as they can exploit the nonlinear correlations between several input variables. The reliability of their results is ensured by checking the input and output distribution between the training samples and the data in various control regions.

The first stage of the analysis process is the online selection. Collision events are collected and recorded via dedicated trigger paths that meet specific physics goals (e.g. high- $P_{T}$ lepton identification). Combining multiple paths maximizes the acceptance, however the trigger efficiency must be parametrized properly on Monte Carlo events. Especially in multiple-objects trigger paths (e.g. $\mathscr{E}_{T}$ plus jets) the efficiency function may depend on many variables. At $\mathrm{CDF}$, for the first time $[4,8]$ we used a $\mathrm{NN}$ to model the probability distribution of events selected by a large set of triggers.

The next stage is the offline event selection. We gained acceptance with the introduction of more lepton categories (track only reconstruction, likelihood and $\mathrm{NN}$ identification, etc.) and relaxing the cuts on jets and $E_{T}^{\prime}$ selection [8, 9]. This increased the multi-jet background but multivariate techniques proved to be extremely effective to reduce it and keep it under control $[6,8,9]$.

The last selection stage is the application of b-tagging algorithms to select jets enriched in heavy flavors. The identification of $b \bar{b}$ events can reduce the $W / Z$ plus jets (of generic flavor) background by a factor of 100 although at the cost of a significant inefficiency on signal $\left(\epsilon_{b-t a g} \lesssim\right.$ $50 \%$ ). CDF and D0 collaborations undertook a strong effort on b-tagging strategy optimization. The CDF analyses combine the response of three different b-taggers: the SECVTX [15] algorithm identifies displaced secondary vertexes, the JETPROB [16] algorithm exploits the impact parameter of the tracks and, finally, the information of a neural-network-based tagger can also be used. The D0 analyses deploy a BDT [17] algorithm that includes information relating to the lifetime of the hadrons in the jet. The result is a continuous variable discriminating between $b$ and light jets.

After the selection is complete, we remain with a $W / Z$ plus heavy flavor jets sample that could contain the Higgs signal. The invariant mass of the di-jet system is, by definition, the variable that distinguish a resonance over a nonresonant background produced by QCD interactions, so, in this case, the final sensitivity is limited by the jet energy resolution. Also other variables with smaller separation power exists, CDF combines them using Neural Networks (up to 7 variables are used) while D0 analyses use BDT (with up to 32 variables). The use of multivariate techniques improves the final sensitivity up to $20 \%$ over the simple invariant mass approach.

\section{2 $H \rightarrow b \bar{b}$ Sensitivity}

The final expected and observed sensitivity of the individual primary search channels for different $M_{H}$ are summarized in Table 1 for CDF and Table 2 for D0. The most sensitive analysis can exclude at $95 \%$ C.L. the presence of a Higgs boson of $M_{H}=115 \mathrm{GeV} / c^{2}$ produced with a cross section of 2.3 times the one predicted by the SM.

Individually none of these analyses reaches the SM sensitivity for any analyzed $M_{H}$, however each experiment 
Table 1. Observed and expected 95\% C.L. measured by the CDF experiment using a luminosity up to $7.8 \mathrm{fb}^{-1}$ for different SM Higgs boson masses in the primary search channels $(\ell \ell+b \bar{b}$, $\ell \ell+b \bar{b}, \ell \ell+b \bar{b})$.

\begin{tabular}{lccccccc}
\hline$M_{H}\left(\mathrm{GeV} / c^{2}\right)$ & 100 & 105 & 110 & 115 & 120 & 125 & 130 \\
\hline$Z H \rightarrow \ell \ell+b \bar{b}$ & & & & & & & \\
Exp. & 2.7 & 3.1 & 3.4 & 3.9 & 4.7 & 5.5 & 7.0 \\
Obs. & 2.8 & 3.3 & 4.4 & 4.8 & 5.4 & 4.9 & 6.6 \\
\hline$W H \rightarrow \ell \not+b \bar{b}$ & & & & & & & \\
Exp. & 1.8 & 2.0 & 2.2 & 2.6 & 3.1 & 3.7 & 4.8 \\
Obs. & 1.1 & 2.1 & 2.8 & 2.7 & 3.4 & 4.4 & 6.1 \\
\hline$V H \rightarrow \not \ell+b \bar{b}$ & & & & & & & \\
Exp. & 2.3 & 2.4 & 2.6 & 2.9 & 3.4 & 4.0 & 4.9 \\
Obs. & 1.8 & 1.8 & 2.2 & 2.3 & 3.3 & 5.4 & 5.0 \\
\hline
\end{tabular}

Table 2. Observed and expected 95\% C.L. measured by the D0 experiment using a luminosity up to $8.5 \mathrm{fb}^{-1}$ for different SM Higgs boson masses in the primary search channels $(\ell \ell+b \bar{b}, \ell \ell+$ $b \bar{b}, \phi k+b \bar{b})$.

\begin{tabular}{lccccccc}
\hline$M_{H}\left(\mathrm{GeV} / c^{2}\right)$ & 100 & 105 & 110 & 115 & 120 & 125 & 130 \\
\hline$Z H \rightarrow \ell \ell+b \bar{b}$ & & & & & & & \\
Exp. & 3.4 & 3.7 & 4.2 & 4.8 & 5.3 & 6.5 & 8.4 \\
Obs. & 2.5 & 2.6 & 3.1 & 4.9 & 6.4 & 8.9 & 9.9 \\
\hline$W H \rightarrow \ell v+b \bar{b}$ & & & & & & & \\
Exp. & 2.4 & 2.6 & 3.0 & 3.5 & 4.3 & 5.4 & 7.0 \\
Obs. & 2.6 & 2.9 & 4.1 & 4.6 & 5.8 & 6.8 & 8.2 \\
\hline$V H \rightarrow \not \ell+b \bar{b}$ & & & & & & & \\
Exp. & 2.8 & 2.9 & 3.1 & 4.0 & 4.5 & 5.4 & 6.9 \\
Obs. & 2.6 & 2.4 & 2.4 & 3.2 & 3.9 & 5.0 & 7.5 \\
\hline
\end{tabular}

can combine the three channels to obtain powerful constraints on the $H \rightarrow b \bar{b}$ production and decay. The combination of different channels, across the same experiment, provides also an advantage in the evaluation of the correlated systematic uncertainties. For example effects like Jet Energy Scale (JES) uncertainty or b-tag efficiency measurement on MC are shared across all the channels and we fit for their best value [18], in this way a higher statistical sample poses a stronger constraint on these systematics than each channel by itself. Figure 4 shows that the CDF experiment by itself excludes, at $95 \%$ C.L., the presence of a Higgs boson for $M_{H}<105 \mathrm{GeV} / c^{2}$, giving an independent confirmation of the LEP [19] exclusion in the same region. The exclusion limit can be extended further combining the results from both the CDF and D0 experiments [20].

\section{Secondary Search Channels}

The primary channels described in the previous sections play a major role in the Higgs boson searches performed by the CDF and D0 collaborations, however there are a variety of final states worth investigating. The most significant are the $H \rightarrow \gamma \gamma[21,22]$, and $H \rightarrow \tau \tau[18,24]$ decay channels and the $t t H \rightarrow l v b \bar{b} b \bar{b}$ associate production [25]. For example, even though the diphoton final state has a tiny branching fraction, it can still contribute significantly

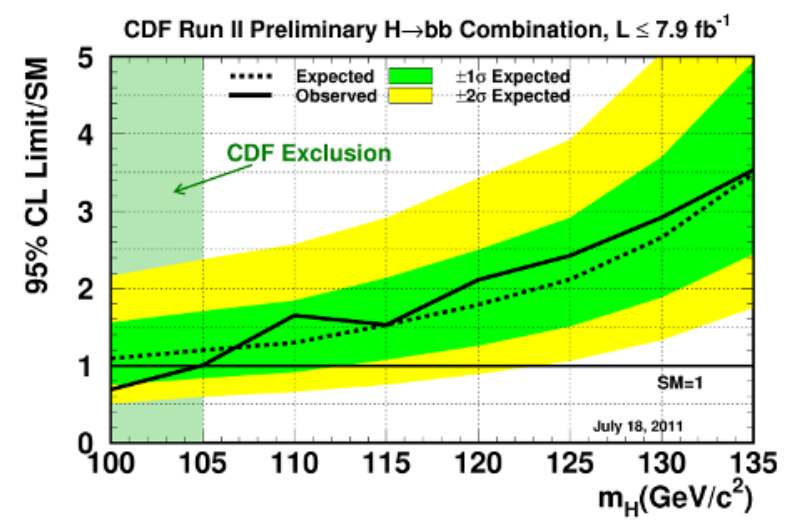

Fig. 3. ble

Fig. 4. Observed and expected $95 \%$ C.L. measured by the CDF experiment using a luminosity up to $7.8 \mathrm{fb}^{-1}$ for different SM Higgs boson masses and with a final state $H \rightarrow b \bar{b}$. The limit is obtained combining all the CDF primary search channels as they share the $H \rightarrow b \bar{b}$ signature.

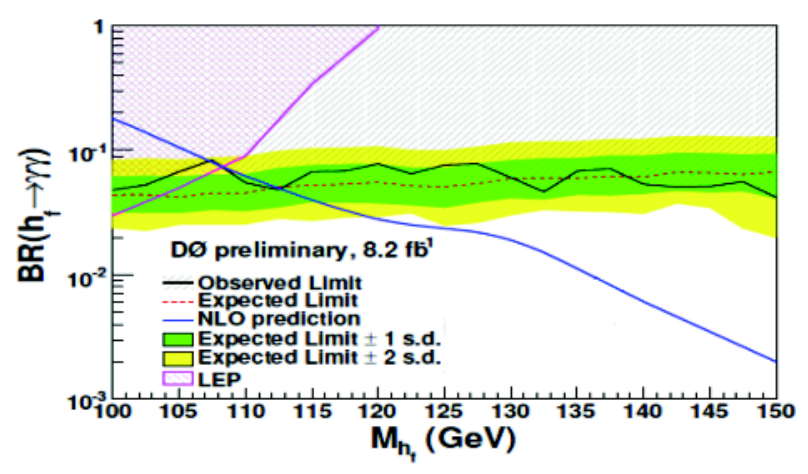

Fig. 5. Observed and expected $95 \%$ C.L. measured by the D0 experiment using a luminosity of $8.2 \mathrm{fb}^{-1}$ in the $H \rightarrow \gamma \gamma$ channel for a fermiophobic Higgs boson of different masses.

to the low mass Higgs boson searches due to better mass resolution and detector acceptance relative to b-quark final states: for $M_{H}=115 \mathrm{GeV} / c^{2}$, expected sensitivity of $11 \times \sigma_{H}^{S M}$ are reached by D0 and $13 \times \sigma_{H}^{S M}$ by CDF. Similar contribution comes from the $H \rightarrow \tau \tau$ channel (expected sensitivity is $12.8 \times \sigma_{H}^{S M}$ for D0 and $12.6 \times \sigma_{H}^{S M}$ for CDF for $M_{H}=115 \mathrm{GeV} / c^{2}$ ) because the branching ratio of the SM Higgs boson to a $\tau$ pair is the second highest (7.6\% at $M_{H}=115 \mathrm{GeV} / c^{2}$ ); the accurate knowledge of the $Z \rightarrow \tau \tau$ process also helps the analysis of this channel. In general each of the secondary channels reach a sensitivity on the order of $12 \times \sigma_{H}^{S M}$ and the composition of all of them contributes to the final search at the level of an additional primary channel $[27,28]$.

Another reason to pursue these secondary channels is that non-SM theories may predict enhanced yield. For example the $H \rightarrow \gamma \gamma$ analyses can be reinterpreted in the light of a fermiophobic Higgs boson theory where the couplings to the fermions are depressed [26]. Figure 5 shows the exclusion limits posed by the D0 collaboration for this particular model. 


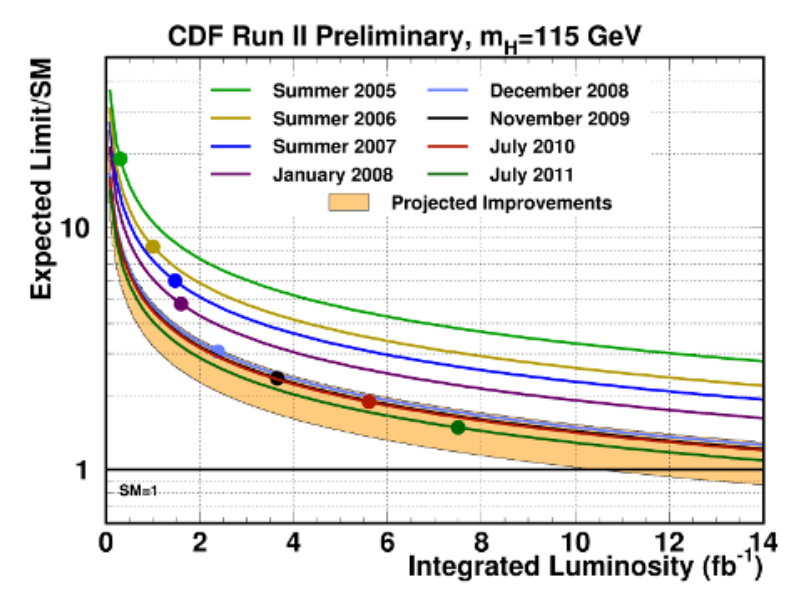

Fig. 6. Plausible scenario of the analysis improvements that can be finalized by the CDF collaboration (similar results are expected from D0) for the Winter 2012 conferences where the full dataset of $10 \mathrm{fb}^{-1}$ will be analyzed.

\section{Results and Future Prospects}

The CDF and D0 experiments performed a variety of searches for a low mass Higgs boson. The favored SM decay channel $(H \rightarrow b \bar{b})$ is analyzed in the associated production modes, $W H$ and $Z H$, where the leptonic decay of the vector boson allows an efficient online selection and offline reconstruction of the candidates. The expected sensitivity, for the best channel and $M_{H}=115 \mathrm{GeV} / c^{2}$, reaches $2.6 \times \sigma_{H}^{S M}$. The $H \rightarrow b \bar{b}$ channels have been combined within each experiment to exclude at $95 \%$ C.L. Higgs boson production for $M_{H}<105 \mathrm{GeV} / c^{2}$.

Also the results of a variety of less favorite search channels is analyzed: $H \rightarrow \gamma \gamma, H \rightarrow \tau \tau, t t H$ associate production. They reach approx $12 \times \sigma_{H}^{S M}$ sensitivity (for $M_{H}=115 \mathrm{GeV} / c^{2}$ ). Furthermore they can strongly constrain exotic models, for example the fermiophobic Higgs model has been excluded (at $95 \%$ C.L.) up to $M_{H}<$ $109 \mathrm{GeV} / c^{2}[21,22]$.

Figure 6 shows a plausible scenario of the analysis improvements that can be finalized by the CDF collaboration (similar results are expected from D0) for the Winter 2012 conferences when the full dataset of $10 \mathrm{fb}^{-1}$ will be analyzed. Thanks to the improvements planned by the CDF and D0 collaborations, we expect that the Tevatron will reach the sensitivity needed to exclude at 95\% C.L. a SM Higgs boson across the mass range $100 \lesssim M_{H} \lesssim$ $135 \mathrm{GeV} / c^{2}[20]$.

\section{Acknowledgments}

We would like to thank the organizers of the 2011 Hadron Collider Physics Symposium for a wonderful conference with excellent presentations and the CDF and D0 collaborations for the results presented at this conference.

\section{References}

1. P.W. Higgs, Phys. Lett. 12132 (1964); idem, Phys. Rev. Lett. 13508 (1964); idem, Phys. Rev. 1451156
(1966); F. Englert and R. Brout, Phys. Rev. Lett. 13 321 (1964); G. S. Guralnik, C. R. Hagen and T. W. B. Kibble, Phys. Rev. Lett. 13585 (1964).

2. S. L. Glashow, Nucl. Phys. 22, 579 (1961); S. Weinberg, Phys. Rev. Lett. 19, 1264 (1967); A. Salam, Elementary Particle Theory, ed. N. Svartholm (Almquist and Wiksells, Stockholm), 367 (1968).

3. M. Baak et al., arXiv:1107.0975v1[hep-ph].

4. The CDF Collaboration, CDF Notes 10593 and 10572.

5. The D0 Collaboration, D0 Note 6166-CONF.

6. The CDF Collaboration, CDF Note 10596.

7. The D0 Collaboration, D0 Note 6220-CONF.

8. The CDF Collaboration, CDF Note 10583.

9. The D0 Collaboration, D0 Note 6219-CONF.

10. T. Aalton et al. [CDF Collaboration], Phys. Rev. Lett. 103, 092002 (2009).

11. V. M. Abazov et al. [D0 Collaboration], Phys. Rev. Lett. 103, 092001 (2009).

12. The CDF Collaboration, CDF Notes 10598.

13. J. F. Grivaz, "Searches for diboson production with heavy-flavor jets in the final state at the Tevatron", this proceeding.

14. C. M. Bishop., "Pattern Recognition and Machine Learning”, Springer (2006). ISBN 0-38-731073-8.

15. Acosta, D.E. et al. [CDF Collaboration], Phys. Rev. D 71 052003, (2005).

16. Abulencia, A. et al. [CDF Collaboration], Phys. Rev. D 74072006 (2006).

17. Abazov V.M et. al., Nucl. Instrum. Meth. A 620, 400 (2010).

18. The CDF Collaboration, CDF Note 10609.

19. LEP Electroweak Working Group, http://lepewwg.web.cern.ch/LEPEWWG/

20. W.M. Yao, "Standard Model Higgs Boson Combination at the Tevatron", this proceeding.

21. T. Aaltonen et al. [CDF Collaboration], Phys. Rev. Lett. 108, 011801 (2012). arXiv: 1109.4427[hep-ex].

22. The D0 Collaboration, D0 Note 6177-CONF.

23. T. Aaltonen et al., [The CDF Collaboration],arXiv: 1201.4880.

24. The D0 Collaboration, arXiv:1106.4555v1 [hep-ex].

25. The CDF Collaboration, CDF Note 10574.

26. A. Barroso, L. Brucher, and R. Santos, Phys. Rev. D 60, 035005 (1999); J. Gunion, R. Vega, and J. Wudka, Phys. Rev. D 42, 1673 (1990); A. G. Akeroyd, Phys. Lett. B 368, 89-95 (1996). [hep$\mathrm{ph} / 9511347]$.

27. The CDF Collaboration, CDF Note 10609.

28. Conference Note D0 Note 6229-CONF. 